\title{
Dissipation in quasistatically driven disordered systems
}

\author{
Jordi Ortín and Jürgen Goicoechea \\ Dept. d'Estructura i Constituents de la Matèria, Facultat de Física, Universitat de Barcelona, Diagonal 647, \\ E-08028 Barcelona, Catalonia, Spain
}

(Received 18 March 1998)

\begin{abstract}
This work presents an analysis of hysteresis and dissipation in quasistatically driven disordered systems. The study is based on the random field Ising model with fluctuationless dynamics. It enables us to sort out the fraction of the energy input by the driving field stored in the system and the fraction dissipated in every step of the transformation. The dissipation is directly related to the occurrence of avalanches, and does not scale with the size of Barkhausen magnetization jumps. In addition, the change in magnetic field between avalanches provides a measure of the energy barriers between consecutive metastable states. [S0163-1829(98)00933-3]
\end{abstract}

\section{INTRODUCTION}

Hysteresis is an ubiquituous phenomenon exhibited by systems of very different nature. Macroscopically, hysteresis shows up as a lag in the response to an external driving, accompanied by a dependence on previous history and by energy dissipation. From a microscopic point of view, hysteresis reflects the presence of multiple metastable configurations accessible to the system.

In the last few years, much of the attention on hysteresis has focused on dynamic effects, arising from a competition between the time scale for relaxation from a nonequilibrium state and the time scale of the driving. ${ }^{1}$ Interestingly, however, many systems display hysteresis even when they are driven exceedingly slowly. Examples can be found in all branches of physics. ${ }^{2}$ It is only recently that quasistatic hysteresis in these systems has been associated with the presence of disorder. ${ }^{3}$ Disordered systems present a complex free energy landscape in configuration space, with multiple local minima separated by large energy barriers. The barriers are so large compared to thermal fluctuations that these systems, on practical time scales, remain trapped in a metastable configuration. They move from one local minimum to another only when the motion is driven by an external field. This motion takes place very far from equilibrium and gives rise to avalanches of the system response, which are found to distribute in a very wide range of sizes. The actual state of the system depends on its overall previous history-which often gives rise to striking memory properties.

In spite of its fundamental interest and technological relevance, a general analysis of the energy balance in these quasistatic hysteretic processes is not yet available. The main difficulties lie in (i) the multiplicity of metastable configurations available, (ii) the dependence of the current state of the system on its previous history, and (iii) the intrinsically dissipative character of the evolution. The energy dissipated can be evaluated without difficulty for a closed cycle, such as that of Fig. 1 (top): a straightforward application of energy conservation shows that the dissipation amounts to the area enclosed by the cycle. ${ }^{4}$ If instead of a closed cycle we consider individual transformation events, however, only a fraction of the energy input by the external field is dissipated irreversibly, and the remainder is stored in the system. We lack a general principle to sort out the two contributions. The importance of the problem has recently been recognized by Bertotti, ${ }^{5}$ who has carried out a study on the Preisach model for hysteresis. Other attempts in this direction ${ }^{6}$ can only be considered partially successful.

In this paper we perform such analysis on a fluctuationless random field Ising model (RFIM). Sethna et al. ${ }^{3}$ have shown that this model provides a realistic picture of the collective and nonequilibrium behavior of the hysteretic systems of interest here. The RFIM with fluctuationless dynamics has provided (i) a physical understanding of the returnpoint memory displayed by partial trajectories (Fig. 1) and (ii) an explanation for the recurrent observation of power laws in the event size distributions of fluctuationless hysteretic systems, in terms of a nonequilibrium phase transition controlled by the statistical distribution of the disorder. ${ }^{7}$ Here, we show that a local energy balance can be written for each magnetization event, leading to explicit separate expressions for the energy stored and the energy dissipated. Our analysis leads also to a measure of the energy barriers between metastable configurations. The results are exact for the fluctuationless RFIM, and relevant in general for driven dissipative systems in which the time scale of transition events is negligible in comparison with the time scale of the driving.

\section{THE RFIM AT $T=0$}

The RFIM is a spin model defined on a regular lattice of $N$ sites in $d$ dimensions. The model incorporates the effect of disorder in the form of magnetic fields $h_{i}$, drawn from a random distribution and quenched on each lattice site $i$. The Hamiltonian of the RFIM in the presence of an external driving field $H$ is given by

$$
\mathcal{H}=-J \sum_{\langle i j\rangle} s_{i} s_{j}-\sum_{i} h_{i} s_{i}-H \sum_{i} s_{i}
$$

where $J$ represents a ferromagnetic interaction $(J>0)$ and $s_{i}$ are Ising spin variables which take values \pm 1 . The notation $\langle i j\rangle$ indicates that the interaction is limited to nearest neighbors. 


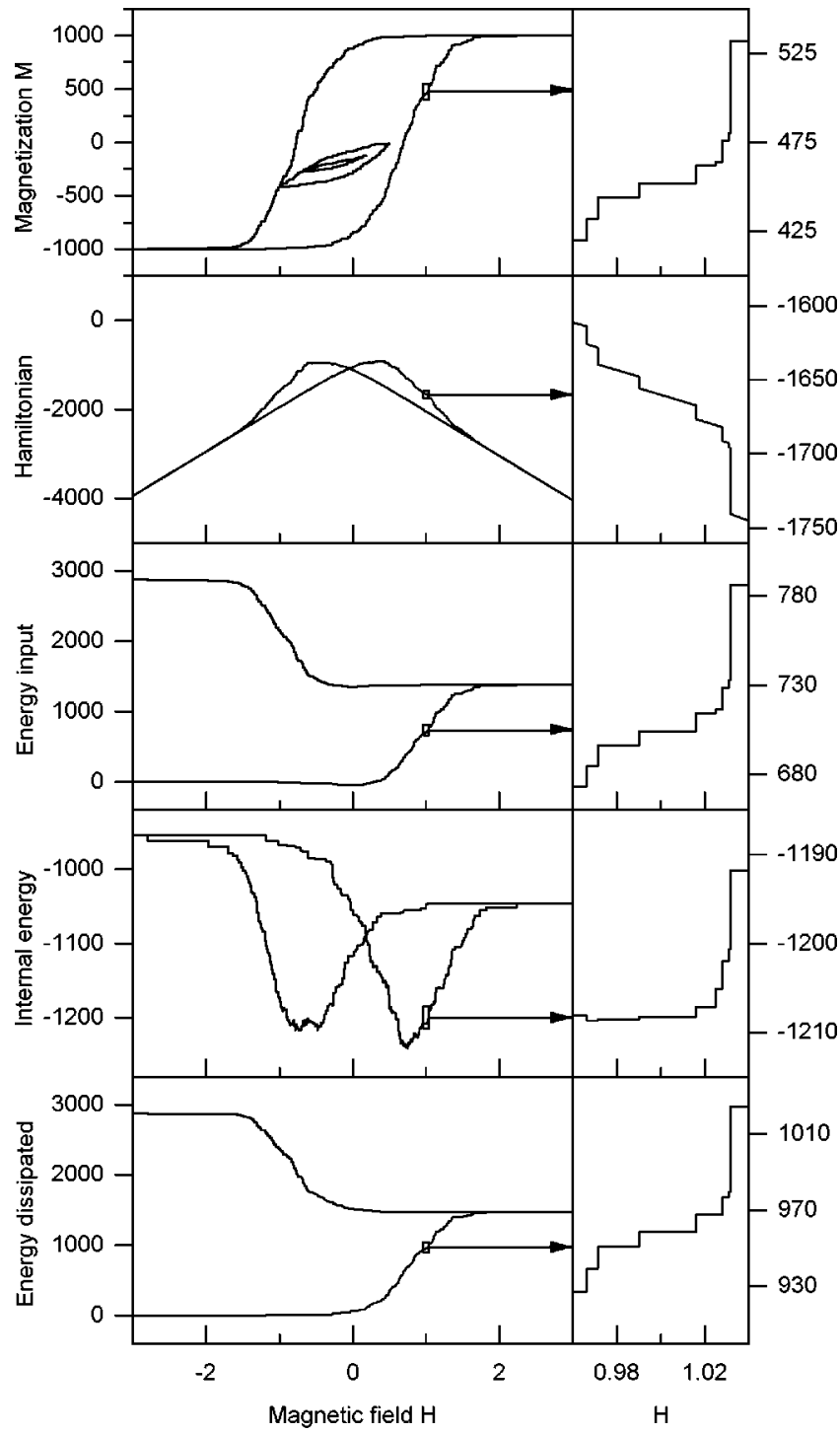

FIG. 1. Top: hysteresis cycle of a fluctuationless RFIM ( $d=1$, $N=1000, J=1$, and Gaussian distribution of the random fields with zero mean and standard deviation $\sigma=1.0$ ), including two internal partial cycles. On the right is a detail of the ascending transformation trajectory. From top to bottom, the evolution of the Hamiltonian, the energy input by the driving field, the internal energy of the system, and the energy dissipated along the cycle, together with a detail of each curve along the ascending trajectory on the right.

The model contains three basic ingredients: a ferromagnetic interaction between neighboring spins, the interaction of every spin with its random field, and the interaction of the spins with the external field driving the transition. The multiplicity of metastable configurations in the system arises from the disorder present.

In the absence of thermal fluctuations the system is governed by synchronous zero-temperature single-spin-flip dynamics: given a value of the field $H$, all the spins in the lattice whose flip decreases $\mathcal{H}$ are flipped simultaneously. Since $\mathcal{H}=\mathcal{H}\left(\left\{s_{i}\right\}, H\right)$, the evolution of the system can be described by ${ }^{8}$

$$
d \mathcal{H}=-\sum_{i}\left(J \sum_{\langle j \mid i\rangle} s_{j}+h_{i}+H\right) d s_{i}-\sum_{i} s_{i} d H
$$

The notation $\langle j \mid i\rangle$ refers to lattice sites $j$ which are nearest neighbors of $i$. The term between brackets is the local magnetic field $F_{i}$ experienced by $s_{i}$.

Given a realization of the disorder $\left\{h_{i}\right\}$, in the absence of thermal fluctuations every spin takes the orientation of its local field. Upon varying $H$ the local fields change correspondingly, but the spin configuration remains unchanged until $F_{i}$ becomes 0 at a given lattice site, making the corresponding spin $s_{i}$ flip at constant $H(d H=0)$ without energy barriers $(d \mathcal{H}=0)$. But the flip of $s_{i}$ modifies the local fields $F_{j}$ experienced by the neighbors: one or more $F_{j}$ may change their sign, making the corresponding spins $j$ flip and $\mathcal{H}$ to decrease. These spins on their turn may trigger new flips of the neighbors, and so on, giving rise to an avalanche of spin flips at constant $H$. The avalanche, and the concomitant Barkhausen jump, arrests when no more spins experience an opposite local field: the system is trapped in a new metastable configuration. To leave this configuration, in the absence of thermal fluctuations, the system must be driven again with $H$ until a new spin experiences a local field $F_{i}$ $=0$ and flips in equilibrium (and eventually triggers a new avalanche of nonequilibrium spin flips at constant $H$ ), and so on. The evolution of the system is thus composed of intervals where $\left\{s_{i}\right\}$ is fixed and the free energy landscape is modified by $H$ and intervals where the landscape does not change and $\left\{s_{i}\right\}$ moves from a metastable state to another at constant $H$. Actually, since an avalanche is the overall result of a sequence of magnetization events at constant $H$ (a sequence of synchronous lattice updatings) the first term in Eq. (2) stands for

$$
\left.d \mathcal{H}\right|_{H}=-\sum_{k} \sum_{i} F_{i}(k) d s_{i}(k)
$$

where the index $k$ runs from 1 to the total number of lattice updatings in the avalanche and $F_{i}(k), s_{i}(k)$ represent the values of $F_{i}$ and $s_{i}$ before the $k$ th lattice updating.

Notice two relevant properties of the dynamics. First, it is deterministic: given a realization $\left\{h_{i}\right\}$, the trajectories followed in configuration space are exactly reproducible. Second, it is adiabatic: the evolution is independent of driving rate. This property corresponds to a complete separation of time scales: avalanches are instantaneous on the time scale of the driving.

The anhysteretic magnetization curve, i.e., the curve going from the equilibrium configuration $\{-1\}$ at $H \ll 0$ to the equilibrium configuration $\{+1\}$ at $H \gg 0$ through a reversible path, connects the configuration of the system which are absolute minimizers of $\mathcal{H}$ for each value of $H$, and for it $\left.d \mathcal{H}\right|_{H}=0$. In the hysteresis cycle of Fig. 1 (top), the anhysteretic falls approximately halfway between forward and reverse magnetization curves. The reason why this curve is not followed by the system is that the lack of fluctuations prevents the system from going over the energy barriers separating the stable equilibrium configurations. The system can only explore a limited portion of configuration space around a given state. As a result, it departs progressively from equilibrium. ${ }^{9}$ 


\section{ENERGY BALANCE}

Depending on the magnetic history of the system, different configurations (and different magnetizations $M$ ) can be attained at a given field $H$. For this reason, the variables $(M, H)$ are not sufficient to describe the nonequilibrium states of the system; instead of $M$, the ordered set $\left\{s_{i}\right\}$ must be used. The configuration $\left\{s_{i}\right\}$ is the proper extension because the return-point memory property ${ }^{3}$ guarantees that $\left\{s_{i}\right\}$ is recovered exactly after a cyclic variation of $H$, so that all functions of $\left\{s_{i}\right\}$ and $H$ are state functions.

Now, to establish an energy balance in the presence of dissipation, we note that in the quasistatic limit the evolution of the system as a function of $H$ is formed by a dense sequence of metastable equilibrium states, because avalanches are instantaneous on the time scale of the driving. For such a quasistatic dissipative evolution at $T=0$, the internal energy of the system $\mathcal{U}$ changes according to $d \mathcal{U}=H d M-\mathbb{d} \Psi$, where $H d M$ is the work input by the driving field and $d \Psi$ is the energy dissipated. The Hamiltonian and the internal energy of the system at zero temperature are related by the Legendre transformation $\mathcal{H}=\mathcal{U}-H M$. Thus, at constant temperature $d \mathcal{H}=-\mathbb{d} \Psi-M d H$. Comparing to $d \mathcal{H}$, given by Eq. (2), we conclude that

$$
\varpi \Psi=\sum_{i} F_{i} d s_{i}
$$

This result identifies the energy dissipation in every spin flip with the product of an unbalanced force (local magnetic field) times a generalized flow (local change in magnetization). Recall that the first flip in an avalanche takes place when $F_{i}=0$, but additional flips triggered by this first flip take place when $F_{i} \neq 0$. Since in these additional flips $F_{i}$ and $d s_{i}$ have the same sign, $d \Psi \geqslant 0$, as expected. Equation (4) shows that energy dissipation takes place only at avalanches, where $F_{i} \neq 0$. This is the physical origin of hysteresis.

According to this result, energy dissipations do not scale with Barkhausen magnetization jumps $d M=\Sigma_{i} d s_{i}$. We have studied the cumulative distributions of these two magnitudes in a two-dimensional (2D) RFIM of size $100 \times 100$, for Gaussian distributions of $\left\{h_{i}\right\}$ with zero mean and standard deviations $\sigma=1.10,0.92$, and 0.70 . Averages have been performed over 100 realizations of the disorder. The result is shown in Fig. 2. While the distribution of magnetization jumps (in the inset) approaches a power law around $\sigma$ $=0.92$, with a cutoff due to finite-size effects, ${ }^{7}$ the distribution of energy dissipations does not show any hint of scale invariance in the range of $\sigma$ explored. Remarkably, however, the distribution of energy dissipations extends over eight decades in energy, in spite of the small system size.

To go one step further in the analysis, we note that $d \mathcal{U}$ $=d \mathcal{H}+H d M+M d H$. Combined with Eq. (2), it leads to

$$
d \mathcal{U}=-\sum_{i}\left(J \sum_{\langle j \mid i\rangle} s_{j}+h_{i}\right) d s_{i} .
$$

This is the change in internal energy of the system in a transformation event. The energy is stored in the interaction energy between spins and between spins and quenched random fields. Given a realization of $\left\{h_{i}\right\}$, Eq. (5) shows that $\mathcal{U}$ de-

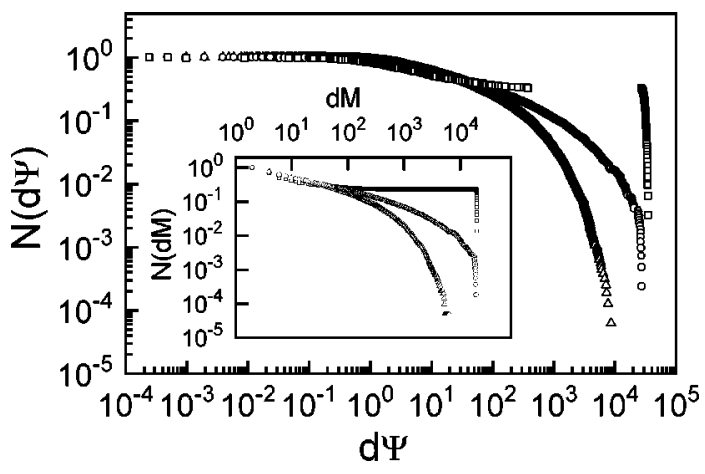

FIG. 2. Cumulative distributions of energy losses, computed along the complete hysteresis cycle of a $100 \times 100$ RFIM, with Gaussian distributions of $\left\{h_{i}\right\}$ with zero mean and standard deviations $\sigma=0.70,0.92$, and 1.10 (from top to bottom). Averages have been taken over 100 realizations of the randomness. The inset shows the corresponding cumulative distributions of Barkhausen jumps.

pends only on $\left\{s_{i}\right\}$, and therefore will return to its original value after a cyclic excursion of $H$.

In conclusion, a balance of the form $H d M=d \mathcal{U}+d \Psi$ applies to each transformation event, reflecting that the energy input by the driving field is partially stored as internal energy and partially dissipated at avalanches. The evolution of these contributions along a closed hysteresis cycle is shown in Fig. 1. Immediately, since $\mathcal{U}$ is a state function, we recover for a closed cycle at constant temperature the result ${ }^{4}$

$$
\oint d \Psi=\oint H d M
$$

This relates the overall dissipation in the hysteresis cycle with the area enclosed by the cycle. Recalling that $d \Psi \geqslant 0$, Eq. (6) shows that $M-H$ cycles can be contoured in one sense but not in the opposite sense.

\section{DISTRIBUTION OF ENERGY BARRIERS}

The distribution of energy barriers along the transformation can be derived from the hysteresis cycle. Let $\Delta \mathcal{H}$ be the height of the energy barrier between any two consecutive metastable configurations, assumed to be reached at fields $H_{1}$ and $H_{2}$, respectively. Then $\Delta \mathcal{H}=\mathcal{H}\left(\left\{s_{i}^{\prime}\right\}, H_{1}\right)$ $-\mathcal{H}\left(\left\{s_{i}\right\}, H_{1}\right)$, where $\left\{s_{i}\right\}$ is the configuration reached at $H_{1}$ and $\left\{s_{i}^{\prime}\right\}$ is the configuration that differs from $\left\{s_{i}\right\}$ in a single spin and either it is reached at $H_{2}$ (if no avalanche occurs) or it triggers the avalanche towards the configuration reached at $\mathrm{H}_{2}$. Taking into account that the spin flipped on going from $\left\{s_{i}\right\}$ to $\left\{s_{i}^{\prime}\right\}$ flips in equilibrium at $H_{2}$, the corresponding local field is 0 and we obtain

$$
\Delta \mathcal{H}=2 s_{i}^{\prime}\left(H_{2}-H_{1}\right) .
$$

This result shows that the field intervals between transformation events provide a direct measure of the energy barriers between the corresponding metastable states. Note that $s_{i}^{\prime}$ $=+1$ when $H_{2}>H_{1}$ and $s_{i}^{\prime}=-1$ when $H_{2}<H_{1}$, so that $\Delta \mathcal{H}>0$ in all cases, as expected. Following this result, we have computed the distributions of energy barriers overcome by the 2D RFIM studied before. The shape of the cumulative 


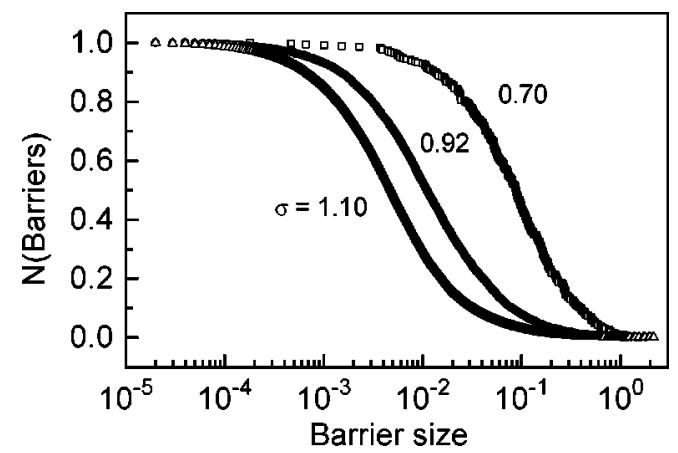

FIG. 3. Cumulative distributions of barrier sizes, encountered along the complete hysteresis cycle of the 2D RFIM considered in Fig. 2, for three different Gaussian distributions of random fields.

distributions in Fig. 3 does not seem to be sensitive to $\sigma$. Remarkably, barrier sizes extend over five decades in energy, making evident the complexity of the free energy landscape.

\section{CONCLUSIONS}

We have considered the phenomenon of quasistatic hysteresis in driven dissipative systems which exhibit a complete separation of time scales. The fluctuationless RFIM has been taken as a paradigm of the collective and far from equilibrium behavior of these systems.

In the fluctuationless RFIM, a cyclic variation of $H$ carries the system back to its original configuration. Thanks to this return-point memory, potentials which depend only on $\left(\left\{s_{i}\right\}, H\right)$ are true state functions. Making use of this result, together with the quasistatic character of the trajectories, we have been able to provide explicit expressions for the different energy contributions at individual transformation events.

Studies of this kind have been carried out previously on the Preisach phenomenological model of hysteresis ${ }^{5}$ and on a model of ferromagnetic hysteresis based on domain-wall motion. ${ }^{10}$ Our study on the RFIM is more realistic because it takes into account interactions between particles.

Avalanches have been identified as the sources of energy dissipation. We have shown that energy losses do not scale with avalanche sizes, and distribute differently along the hysteresis cycle. We have shown also that the field intervals between transformation events provide a direct measure of the energy barriers separating metastable configurations.

The systematics developed in this work can be applied quite generally to deterministic models of driven dissipative systems in the limit where the driving is carried out infinitely slowly. Examples include cellular-automata models of sandpiles (see Ref. 2), and models of fluid invasion in porous media. ${ }^{11}$ Our results may also be used to extract physical information from experimental data on these and related systems.

\section{ACKNOWLEDGMENTS}

We thank E. Vives, A. Planes, and Ll. Carrillo for interesting discussions. This work received financial support from the Comisión Interministerial de Ciencia y Tecnología (CICyT, Spain), Project No. MAT95-0504, and the Dirección General de Enseñanza Superior (DGES, Spain), Project No. PB96-0378-C02-01.
${ }^{1}$ M. Acharyya and B. K. Chakrabarti, Phys. Rev. B 52, 6550 (1995); Z. Fan and Z. Jinxiu, Phys. Rev. Lett. 75, 2027 (1995), and references therein.

${ }^{2}$ In ferromagnets, D. C. Jiles, and D. L. Atherton, J. Appl. Phys. 55, 2115 (1984); D. C. Jiles, Introduction to Magnetism and Magnetic Materials (Chapman and Hall, New York, 1991). In soil-water sorption and desorption processes, D. Hillel, Soil and Water: Physical Principles and Processes (Academic, New York, 1971), and references therein. In capillary-condensation, M. P. Lilly, P. T. Finley, and R. B. Hallock, Phys. Rev. Lett. 71, 4186 (1993). In charge-density waves, Z. Z. Wang and N. P. Ong, Phys. Rev. B 34, 5967 (1986). In superconductors, S. Field, J. Witt, F. Nori, and X. Ling, Phys. Rev. Lett. 74, 1206 (1995). In structural transitions, J. L. McNichols, Jr. and J. S. Cory, J. Appl. Phys. 61, 972 (1987); J. Ortín, ibid. 71, 1454 (1992), and references therein. In cellular automata models of sandpiles, J. Goicoechea and J. Ortín, Phys. Rev. Lett. 72, 2203 (1994).

${ }^{3}$ J. P. Sethna, K. Dahmen, S. Kartha, J. A. Krumhansl, B. W. Roberts, and J. D. Shore, Phys. Rev. Lett. 70, 3347 (1993).
${ }^{4}$ T. Iwata, J. Magn. Magn. Mater. 59, 215 (1986).

${ }^{5}$ G. Bertotti, Phys. Rev. Lett. 76, 1739 (1996).

${ }^{6}$ J. S. Cory and J. L. McNichols, Jr., J. Appl. Phys. 58, 3282 (1985); Y. Huo and I. Muller, Continuum Mech. Thermodyn. 5, 163 (1993), and references therein.

${ }^{7}$ K. Dahmen and J. P. Sethna, Phys. Rev. B 53, 14872 (1996), and references therein; J. B. Tadic, Phys. Rev. Lett. 77, 3843 (1996); E. Vives, J. Goicoechea, J. Ortín, and A. Planes, Phys. Rev. E 52, R5 (1995).

${ }^{8} s_{i}$ is a discrete variable which takes values \pm 1 . A derivative with respect to $s_{i}$ is to be understood as the formal result of flipping $s_{i}$ from one value to another. Equivalently, $d s_{i}= \pm 2$.

${ }^{9}$ Exceptionally, for a very broad distribution of $\left\{h_{i}\right\}$, spin flips become completely uncorrelated and avalanches disappear. In this limit the system is not dissipative: forward and reverse magnetization curves converge to a single, reversible, anhysteretic curve.

${ }^{10}$ D. C. Jiles and D. L. Atherton, J. Magn. Magn. Mater. 61, 48 (1986).

${ }^{11}$ R. A. Guyer and K. R. McCall, Phys. Rev. B 54, 18 (1996). 